\title{
Microbiome Structure in Biofilms From a Volcanic Island in Maritime Antarctica Investigated by Genome-Centric Metagenomics and Metatranscriptomics
}

Victor Borin Centurion ( $\nabla$ vborincenturion@gmail.com )

State University of Campinas: Universidade Estadual de Campinas https://orcid.org/0000-0002-55978554

\section{Stefano Campanaro}

University of Padova: Universita degli Studi di Padova

Arianna Basile

University of Padova: Universita degli Studi di Padova

\section{Laura Treu}

University of Padova: Universita degli Studi di Padova

Valéria Maia Oliveira

State University of Campinas: Universidade Estadual de Campinas

\section{Research}

Keywords: genome-centric metagenomics, Whalers Bay, Correlation Network, Purple Bacteria, Stress response

Posted Date: January 21st, 2021

DOI: https://doi.org/10.21203/rs.3.rs-151020/v1

License: (c) (i) This work is licensed under a Creative Commons Attribution 4.0 International License. Read Full License 


\section{Abstract}

\section{Background}

Antarctica is the coldest and driest continent on Earth, characterized by polyextreme environmental conditions, where species adapted to grow and thrive at low values of temperature, low nutrient concentration and relative humidity and high UV radiation form complex networks of interactions. Microbial communities growing in these harsh environments can form biofilms that help the associated species to survive in such harsh conditions. The knowledge on biofilms microbial community in the literature is extensive, however, most studies are focused on the dominant species and are not able to disclose the whole functional complexity and entire metabolic potential.

Methods

To overcome these limitations, the present study used genome-centric metagenomics and metatranscriptomics to analyze two biofilm samples collected in Deception Island, Maritime Antarctica. Species abundance and their functional activity were correlated with environmental parameters. Differential gene expression analysis based on the negative binomial distribution of the DeSeq2 package was used to compare the abundance of metagenome-assembled genome (MAG) in the biofilms (BR and BG) and the gene expression of the metatranscriptomic analysis ( $p$-value 0.05).

Results

The results showed a complex microbiome represented by 180 MAGs, highly divergent according to temperature, $\mathrm{pH}$, and position along a transect of Whalers Bay sediments. Metabolic evaluations allowed to predict polyphenols and chitin as primary substrate feeding. The potential metabolic interactions were investigated using metabolic flux balance analysis and revealed that purple bacteria are the taxonomic group with the highest number of correlations with other bacteria. This behavior is well represented by the MAG WB_595 belonging to Alphaproteobacteria.

\section{Conclusions}

Due to predicted mixothrophic behavior of Alaproteobacteria_WB_595 MAG, it may represent a crucial member of the microbiome, and it has the potential to support the heterotrophic species in the biofilms. Metatranscriptomics revealed that the chaperone system and proteins counteracting reactive oxygen species and toxic compounds have a key role in the maintenance of bacterial cell homeostasis in sediments of volcanic origin.

\section{Introduction}

The advent of innovative molecular techniques such as next generation sequencing introduced deep modification in the study of subjects such as evolution, endemism, invasion, and microbial selection in the Antarctic continent (Vincent, 2000; Bej et al., 2009; Rogers et al., 2013; Richter et al., 2014). 
Metagenomics allows the identification of functional genes directly from environmental samples, and it can disclose the complexity of microbial communities, estimating the associated diversity, understanding the population dynamics of a community and allowing to recover complete genomes of organisms not cultured yet (Rajendhran and Gunasekaran, 2008). Although metagenomics can reveal the genetic potential available in the microbiome, to understand the roles that members of a community are playing and how they respond to environmental variations, it is necessary to integrate metagenomic data with other "omics" approaches, such as metatranscriptomics. Evaluation of gene expression allows classification, identification and quantification of specific responses to environmental stimuli, enabling the establishment of correlations of microbial functions with environmental data (Toseland 64 et al., 2014; Delforno et al., 2019).

Despite the important findings obtained in the past few years, knowledge regarding the microbial roles and interactions in most ecological niches in Antarctic continent is still scarce. Biogeochemical cycles and food chains, in an environment with such restrictive abiotic characteristics, are often formed exclusively by microorganisms, which play a fundamental role in energy transformation, recycling of organic matter, and mineralization of nutrients, forming the basis for the functioning of terrestrial and aquatic ecosystems (Vincent 2000; Yergeau and Kowalchuk, 2008). Microorganisms thriving in this extreme region show an enormous metabolic diversity that allowed them to efficiently colonize the different niches available (Margesin and Miteva, 2011). The environmental conditions in Antarctica are not homogeneous, in fact, there are strong variations in different niches, including different types of soils, sediments, rocks, as well as snow and ice. These factors can vary in the thermal, nutritional, water activity and salinity properties. Notably, the volcanic island Deception, located in Maritime Antarctica, due to geothermal activity, permanent ice, and different types of rocks (Bartolini et al., 2014; Centurion et al., 2019; Geyer et al., 2019), has attracted attention and represents a great target to investigate metabolic adaptation and taxonomic composition of the species growing in this habitat (Bendia et al., 2018a, 2018b). For example, in Whalers Bay, the first landing area of Deception island, constant cold temperatures are often accompanied by freeze/thaw cycles occurring between winter and summer; this results in modifications of the permanent ice cover from year to year (Centurion et al., 2021).

One of the strategies allowing successful growth and protection of the microorganisms in harsh environments is the formation of biofilms, a process which occurs through the production of a dense mass of extracellular polymeric substances (EPS) which involves the microbial cells. Apart from the wellknown cryoprotectant function, EPS can assist microorganisms in scavenging nutrients and metals from the environment and act as a site to accumulate UV-protective enzymes and compounds such as superoxide dismutase and pigments, respectively (Pereira et al., 2009; Anesio et al., 2017). The biofilm not only benefits bacteria, but can also create protected microenvironmental niches characterized by low stressful conditions enabling the growth of some microalgae and mosses that are usually inhibited when exposed directly to restrictive environmental conditions (Rossi and De Philippis, 2015). In Antarctica, prokaryotes can form different biofilm organizations according to the growth place, such as cryoconite granules and microbial mats. Cryoconite is a dark granule mixture characterized by carbon source in the external part and inorganic matter in the internal one; these structures are formed by aeolian debris which 
melt on the ice surface (Smith et al., 2016; Anesio et al., 2017). Microbial mats are colonies formed by associations of microorganisms with lithic surfaces (rocks) characterized by a laminated, multilayered structure (Rossi and Philippis, 2015). The phylum Cyanobacteria in particular, is crucial for the formation and maintenance of biofilms. However, studies performed using metagenomic shotgun or amplicon (16S rRNA gene) sequencing showed scarce presence of Cyanobacteria in biofilms (Cameron et al., 2012; Alvarenga et al., 2017; Anesio et al., 2017). Therefore, studies of biofilms focused on the characterization of microorganisms with the highest abundances may underestimate the importance of rare taxa having crucial roles for the environment. For this reason, the main aim of the current study was to correlate the genomic features of the species present in two biofilms exposed to different stressful conditions in Whalers Bay, Deception Island, in the Maritime Antarctica. In order to reveal the metabolic mechanisms of adaptation, state of the art bioinformatics tools and metatranscriptomic analysis have been used along with an innovative approach to estimate metabolic interactions between microbial species.

\section{Material And Methods}

\section{Sampling}

Sampling was carried out from sediments covered with a red biofilm formed under a glacier and a green biofilm formed on the surface of melting water (Fig. 1a), in Whalers Bay (WB), Deception Island, South Shetlands archipelago (Maritime Antarctica; - S $62^{\circ} 97$ '934' 'and W 060 $55^{\circ} 532$ "), in December 2014, 2015 and 2017 (austral summer). Sediment samples were collected in duplicate (2014) and triplicate (2015 and 2017) using sterilized bags for chemical analysis, Falcon tubes for DNA extraction, and Falcon tubes with LifeGuard Soil Preservation Solution (Qiagen, Inc, Hilden, Germany) for RNA extraction and stored at $-20^{\circ} \mathrm{C}$ for transportation to the laboratory of the Microbial Resources Division (CPQBA / UNICAMP), in Campinas, SP, Brazil. Sample sites of each year comprised the Red biofilm (BR), which spanned site 1 (WB1), subglacial sediments, and site 2 (WB2), $50 \mathrm{~cm}$ far from the glacier; and the Green Biofilm (BG), which spanned site 3 (WB3), central area and site 4 (WB4), transect end (Fig. 1b). At the sampling site, sediment and environmental temperature data were collected. After arrival at the laboratory, sediment samples from each summer were immediately processed for DNA and RNA (only 2017) extraction and sequencing.

\section{Physicochemical Analyses of WB Sediments}

Physicochemical analyses of samples corresponding to each site of the WB sediments of the three sampling years were carried out at the Soil Science Department of Luiz de Queiróz School of Agriculture (ESALQ - USP, Piracicaba, SP). Concentrations of total $\mathrm{N}$ and inorganic $\mathrm{N}\left(\mathrm{N}^{-\mathrm{NH}^{+}}{ }^{4}\right.$ and $\left.\mathrm{N}^{-\mathrm{NO}^{-3}}\right)$ were evaluated by the Kjeldahl test and steam distillation, respectively. The organic carbon levels were evaluated by titration of excess dichromate ions with $\mathrm{Fe}^{+2}$ ions and colorimetric methods based on the reading of green color of $\mathrm{Cr}$ (III) ion reduced by organic carbon (Quaggio and Raij, 1979) with detection limit of $0.00 \mathrm{mg} / \mathrm{dm}^{3}$. The concentration of organic matter was obtained from organic carbon results by 
conversion using the Van Bemmelen factor. Concentrations of the elements $\mathrm{Fe}, \mathrm{Cu}, \mathrm{Mn}, \mathrm{Zn}, \mathrm{Pb}, \mathrm{Cd}, \mathrm{Cr}, \mathrm{Ni}$, and Co were determined by flame atomic absorption spectroscopy.

\section{DNA/RNA extraction and sequencing}

Metagenomic DNA of samples collected from the sediments of Whalers Bay along the three summers was extracted with DNeasy PowerSoil Kit (Qiagen, Inc, Hilden, Germany), according to the manufacturer's instructions. Total DNA obtained from 2014 (duplicate) and 2015 (triplicate) samples were sent for largescale sequencing using the Illumina HiSeq platform ( 2 x $150 \mathrm{bp}$, paired-end) at the MR DNA company (Texas, United States). Samples of the year 2017 were sent to the company GenOne Soluções em Biotecnologia (Rio de Janeiro, RJ, Brazil) for sequencing with Illumina NovaSeq 6000 platform (2 x 150, paired-end). Metagenomic sequences were submitted to the European Nucleotide Archive (http://www.ebi.ac.uk) under the project accession number PRJEB29861 for 2014 samples and PRJEB38669 for 2015 and 2017 samples.

Metatranscriptomic RNA of the year 2017 (triplicate) was extracted with RNeasy PowerSoil Total RNA kit (Qiagen, Inc, Hilden, Germany) according to the manufacturer's instructions. Ribosomal RNA of the samples was depleted using the Ribo-Zero Plus rRNA Kit (Illumina, Inc, California, EUA), according to the manufacturer's instructions. The cDNA synthesis and sequencing in Illumina HiSeq platform (2 x $101 \mathrm{bp}$, paired-end) were performed at the Genomics Center of Luiz de Queiróz School of Agriculture (ESALQ USP, Piracicaba, SP). Metatranscriptomic sequences were submitted to the European Nucleotide Archive (http://www.ebi.ac.uk) under the project accession number PRJEB42062.

\section{Bioinformatics Analyses of Metagenomes}

The quality of the raw sequence data was verified using the fastqc tool (https://www.bioinformatics.babraham.ac.uk/projects/fastqc/). Reads were filtered by the Trimmomatic v 0.39-1 tool (Bolger et al., 2014) to remove adapters and low-quality bases (Phred score $\leq 20$ ); an additional check was performed to remove the bacteriophage phi x174 contamination by BBDuck of BBTools (https://jgi.doe.gov/data-and-tools/bbtools/). The high-quality reads were assembled by using all the 29 paired-end libraries (co-assembly) with the MegaHit v1.2.4 tool (Li et al., 2015). To generate the depth file needed for the binning approach, bowtie2 v2.2.6 (Langmead and Salzberg, 2012) and SAMTool v0.1.19 (Li et al., 2009) were used. The binning process was performed by using five tools: BinSanity v0.3.4-0 (Graham et al., 2017), Concoct v1.1.0-0 (Alneber et al., 2014), Maxbin2 v2.2.6 (Wu et al., 2015), MetaBAT v0.32.5 (Kang et al., 2015) and MetaBAT2 v2.12.1 (Kang et al., 2020). All binning results were dereplicated and aggregated by the DASTool v1.1.2-0 (Sieber et al., 2018). CheckM v1.1.2-1 (Parks et al., 2015) was used to assess quality (completeness and contamination) of the MAGs obtained with each binning tool and to determine the coverage of each sample library in the MAGs of aggregated DASTool results. The taxonomic classification of the MAGs was made by GTDB-Tk v1.0.2 (Chaumeil et al., 2019) and the phylogenetic profile obtained by PhyloPhIAn v3.0.51 (Asnicar et al., 2020). The phylogenetic tree was visualized using iTOL v5 (Letunic and Bork, 2006). The prediction of ORFs (Open Reading Frames) in each MAG was run in Prodigal v2.6.3 tool (Hyatt et al., 2010) and the ORF functional classification in 
DRAM v1.1.1 (Shaffer et al., 2020) and EggNOG v5.0 tools (Huerta-Cepas et al., 2019). Identifiers were assigned to MAGs according to the highest taxonomic level obtained from the analysis, the sampling site name and the number attributed by binning tools.

\section{Bioinformatics Analyses of Metatranscriptomes}

The bowtie2 v2.2.6 tool was used to map high-quality reads (Phred score $\geq 30$ ) of 12 metatranscriptomic libraries against ORFs on the high/intermediate-quality MAGs ( $\geq 90 \%$ completeness, $\leq 5 \%$ contamination and $\geq 70 \%$ completeness, $\leq 10 \%$ contamination, respectively). With this procedure, only functional gene information of each MAG was aligned, excluding the possibility of mapping ribosomal sequences not removed by the depletion kit. To extract the quantitative information of reads mapped of each library, the SAMTool v0.1.19 was used. Finally, the functional classification of ORFs was obtained comparing the ORF codes of the bowtie2 results with the eggNOG tables derived from metagenomic analyses.

\section{Statistical Analysis}

For the physicochemical information, the values were separated in two groups: BR (WB1 and WB2) and BG (WB3 and WB4). Then, the average and standard deviation were calculated and compared using the Ttest. The correlation between physicochemical and microbial data was performed by the function "BIOENV" of the package Vegan v2.5-6 using Spearman, Bray-Curtis and Euclidean coefficients (Clarke and Ainsworth, 1993).

Comparison between WB and Tara Ocean MAGs (Delmond et al., 2018) was made by the calculation of ANI values (Average Nucleotide Identity) in the dRep v2.6.2 tool (OIm et al., 2017). The correlation analysis of the high/intermediate-quality MAGs was made by the FastSpar tool (Watts et al., 2018), which uses the linear Pearson correlations between the log-transformed components from SparCC strategy (Friedman and Alm, 2012). The positive correlation values with p-value 0.01 were uploaded in Cytoscape (Shannon et al., 2003) to create a network, and a cut off of 20 degrees was applied to visualize MAGs with higher number of correlations. For the flux balance analyses, CarveMe v1.2.1 tool (Machado et al., 2018) was used to create the metabolic models of the MAGs. Afterwards, Smetana v1.0.0 tool (Zelezniak et al., 2015) was used for the calculation of the cross-feeding interactions occurring inside the whole microbiome and comparing the two biofilms as previous described (Basile et al., 2020). The Smetana values are the combination of three scores: species coupling score (SCS), metabolite uptake score (MUS) and metabolite production score (MPS). The results of Smetana analyses were summed and transformed in percentage for each biofilm.

Following a procedure previously optimized, differential gene expression analysis based on the negative binomial distribution of the DeSeq2 package (Love et al., 2014) was used to compare the abundance of MAGs in the biofilms (BR and BG) and the gene expression of the metatranscriptomic analysis ( $p$-value 0.05) (Fontana et al., 2018). For better visualization of the metatranscriptomic gene ontology, all annotation of the MAGs and the expressed ORFs of the GO terms of Gene Ontology database (Ashburner et al., 2000; The Gene Ontology Consortium, 2018) were exported to the R Statistical Environment platform for running an enrichment analysis using the topGO tool (Alexa and Rahnenfuhrer, 2020). 


\section{Results And Discussion}

\section{Characteristics of Biofilms}

The subglacial biofilm (BR) is protected from solar radiation and shows low temperature $\left(0-2^{\circ} \mathrm{C}\right)$, while the $B G$ is exposed to higher solar radiation and higher temperature (up to $13^{\circ} \mathrm{C}$ ). The statistical analyses (t-test) of the temperature values corroborated the significant difference (Fig. 1d, p-value 0.00008), confirming this is the most important abiotic factor influencing the biofilm ecosystem. Another critical difference between the two samples is the $\mathrm{pH}$ ( $\mathrm{p}$-value 0.0003), which was close to neutral (7.2) in BR and more acidic (6.3) in BG. The heavy metal concentration in sediments was constant in all sites, only $\mathrm{Cu}$ showed a small difference ( $p$-value of 0.09), with a higher concentration in BR.

The differences related to the physicochemical parameters registered at the two sampling sites likely shaped microbiome composition and functions resulting in different colors of the biofilms, which are potentially associated to the ability of counteracting specific chemical and physical stresses. In general, red and green colors are characteristic of biofilms associated with algal genera. Chlamydomonas and Chloromonas are carotenoid-rich taxa in resting phases and typical determinants of red mass blooms (Horam and Duval, 2001; Remias, 2012). The green color is usually caused by the chlorophyll-rich trophic phase of Chloromonas (Remias, 2012; Anesio et al., 2017). Moreover, the color can also be associated with purple-green phototrophic bacteria and determined by the production of bacteriochlorophyll and carotenoid pigments (Downes et al., 1993; Keely, 2006). The resulting different colors may thus be due to the exposure of distinct levels of UV-radiation and/or to the growth of specific microorganisms at lower or higher temperature. In fact, as previously reported (Centurion et al., 2019), the resistome can vary among Deception island sites, and abiotic factors, such as $\mathrm{Cu}$ and $\mathrm{Zn}$ concentrations, have a significant role in shaping the microbial profiles.

\section{Taxonomy and diversity of Metagenome-Assembled Genomes}

Metagenomic binning of marine microbiomes is usually complex due to the high number of co-occurring species and strains. In order to solve this conundrum, the metagenomic approach was performed using five different tools, followed by dereplication and combination of the microbial genomes. The assembly and binning of the 29 paired-end metagenomic libraries ( $~ 800$ million reads) allowed the identification of 488 non-redundant metagenome-assembled genomes (MAGs): 63 having medium quality ( $\geq 50 \%$ completeness, $\leq 10 \%$ contamination), 116 intermediate quality ( $\geq 70 \%$ completeness, $\leq 10 \%$ contamination) and 57 high quality ( $\geq 90 \%$ completeness, $\leq 5 \%$ contamination). The last two groups were selected for further analyses and discussion (Supplementary Table 1). None of the MAGs were associated to algal or archaeal species. Due to the dense cell wall characteristics, lysis of algae may pose difficulties and this can result in a low coverage value for this group (data not shown). The 180 selected MAGs belonged to the domain Bacteria and in the mapping analysis they recruited from 11 to $33 \%$ of the high-quality reads, depending on the sample (Supplementary Table 2). Taxonomic analysis of MAGs 
revealed that most of them belonged to the phyla Proteobacteria $(n=69)$ and Bacteroidetes $(n=49)$. This finding is corroborated by previous studies performed on Deception Island (Bendia et al., 2020, Bendia et al., 2018a; Bendia et al., 2018b; Centurion et al., 2019; Centurion et al., 2021). The number of MAGs associated to other phyla was lower and included Actinobacteria $(n=27)$, Firmicutes $(n=17)$, Verrucomicrobia $(n=4)$, Cyanobacteria $(n=3)$, Acidobacteria $(n=3)$, Gemmatimonadetes $(n=3)$, and Chloroflexi $(n=1)$ (Fig. 2). Classification at deeper taxonomic ranks revealed that the MAGs were distributed in 91 genera and 40 families, allowing a better characterization of the bacterial diversity in the biofilms. Only two MAGs were not classified at a taxonomic rank higher than Kingdom level. Of all MAGs recovered, only one was identified at the species level, namely the psychrotrophic and chitin-utilizing Arthrobacter psychrochitiniphilus (Wang et al., 2009). This finding clearly evidenced the high level of novelty in the genomes recovered and confirmed that most of the species residing in the environmental biofilms in Antarctica are still completely unknown. Despite the low percentage of organic matter present in the sediment samples $(0.20-0.30 \%)$, the taxonomic assignment at genus level suggested that 70 MAGs are heterotrophic. Only six MAGs were considered oligotrophic and seven autotrophic. Members of the phylum Cyanobacteria $(n=3)$ are responsible for carbon fixation. Moreover, MAGs of the genus Pseudanabaena (family Oscillatoriaceae; phylum Cyanobacteria) are probably involved in the production of cryoconite granules (Uetake et al., 2016; Buda et al., 2020). These granules have organic matter sources in the outer part and minerals in the inner part, and thus can support the growth of heterotrophic organisms. Because of the characteristics of WB samples (melting water and darkish sediment), it is likely that the biofilm was associated with cryoconite granules.

According to literature, most of the microorganisms found in WB are related to samples of marine origin. For example, Psychrobacter and Flavobacterium, the most abundant genera found in several metagenomic datasets (Centurion et al., 2019; Centurion et al., 2021), include strains that are known fish pathogens (González et al., 2000; Chen et al., 2017) and others that were isolated from marine algae and water (Onishchenko and Kiprianova, 2004; Miyashita et al., 2010; Park et al., 2015). For this reason, the WB MAGs $(n=180)$ were compared with those recently recovered from the TARA-Ocean project $(n=2,683$; Delmont et al., 2018). None of the TARA-Ocean MAGs were similar to the WB MAGs (95\% ANI cutoff), revealing that there are no common species between these samples. Most of the WB MAGs showed 75\% ANI to the TARA-Ocean MAGs (Supplementary Fig. 1). Additionally, an extensive pairwise comparison among the WB MAGs revealed that they are not closely associated, and ANI values were always lower than $80 \%$ even between MAGs of the same genus, indicating that the species are not even closely related. This finding suggests that, despite the recovered MAGs are of marine origin, the enormous number of species in the ocean environment, which are still unknown at genome level, deserves a larger effort to gain a comprehensive knowledge on the global marine microbiome.

\section{Metabolic Potential of Whalers Bay Microbial Biofilms}

Functional annotation revealed that, in general, the most abundant metabolisms associated to carbon sources in MAGs were "chitin backbone and oligo cleavage" and "polyphenolics cleavage" (Supplementary Fig. 2). Chitin is the second most abundant biopolymer in nature, and it is present in 
fungi, algae, and in the exoskeletons of insects and crustaceans (Lonhienne et al., 2001). In Antarctica, the primary sources of chitin are crustaceans and algae, and a minor fraction derives from fungi associated with lichens and wood, introduced in Deception Island by human activity (Duarte et al., 2019). Previous study reported that chitinases identified in some Antarctic species of the Arthrobactergenus can efficiently operate at low temperatures (Lonhienne et al., 2001).

Polyphenolic compounds have multiple phenolic functionalities and are secondary metabolites with antioxidant properties, known to be produced by plants. They are also components of fossil fuels and common anthropogenic pollutants. Although Antarctica is known as a pristine continent, bacteria isolated from this environment were found to have the ability to degrade phenol (Lee et al., 2017; Subramaniam et al., 2020). As an example, bacteria (particularly Polaromonas) associated to diesel spill in Carlini Station, Antarctica, have showed the potential to metabolize pollutants (Vásquez et al., 2017). In fact, Polaromonas has been reported in several studies as a species involved in hydrocarbon degradation (Jeon et al., 2004; Saul et al., 2005; Mattes et al., 2008). Characterization of the bacterial resistome in Deception Island ranked the biocide resistance genes (those counteracting the negative effects of phenolic compounds) as the second most abundant class of resistance genes, both in Polaromonas and Psychrobacter (Centurion et al., 2019). These two genera are dominant members of the Deception sediments (Centurion et al., 2019; Centurion et al., 2021) and in the present work 5 and 1 MAG have been associated to Polaromonas and Psychrobacter, respectively. Furthermore, the arsC gene for arsenate reduction and arsenic resistance (Pal et al., 2014), already reported in a resistome study (Centurion et al., 2019), was found as a common function in the MAGs.

Regarding nitrogen metabolism, most of the MAGs are nitrite-oxidizing bacteria (NOB) and denitrifiers. More specifically, all enzymes involved in denitrification process are encoded in the genomes of MAGs Bacteroidetes WB_211, Methylotenera spp. (WB_221, and WB_229) and Oblitimonas WB_231. Interestingly, all MAGs of the genus Methylotenera, which has been previously associated to methylotrophic, ammonia nitrification, and denitrification metabolisms (Schramm et al., 1998), did not show genes for ammonia metabolism. Moreover, the microbiome of Deception sediments includes members of autotrophic (e.g. Pseudonabaena and Rodhoferax), heterotrophic (e.g. Hydrogenophaga and Polaromonas) and diazotrophic genera (Centurion et al., 2021). However, in this study annotation did not allow to identify the nitrogen fixation nifH gene (nitrogenase iron protein) in any of the MAGs. Previous sequenced-based studies from Antarctic metagenomic samples reported the absence of known proteins involved in nitrogen fixation, and suggested the existence of processes using alternative nitrogen sources (Van Goethem and Cowan, 2019).

The Cyanobacteria Pseudonabaena MAGs ( $\mathrm{n}=2$ ) showed two different photosynthetic systems, one encoded by $p s a A-F$ and $p s b A$ genes and the other by $p s b D$. Furthermore, one MAG of the genus Flavobacterium contained genes for photosynthesis. To the best of our knowledge, this is the first report describing autotrophic species within the Flavobacterium genus. One previous study from Lami et al. (2009) reported the presence of the protein proteorhodopsin in the class Flavobacteriia, a transmembrane light-driven proton pump using light and organic compounds as energy sources. Horizontal gene transfer 
events (HGT) from blue-green algae to non-phototrophic bacteria are a natural occurring process, and it is also possible in biofilms (Lee, 2019). In fact, Flavobacterium strains have been found in association with algae (Miyashita et al., 2010; Park et al., 2015).

To show not only the MAGs functional potential to degrade compounds, but also the metabolic exchange and interaction, the flux balance analysis was applied. The results of flux balance analysis

(Supplementary Table 3) showed a cross-feeding interaction mainly based on $\mathrm{Cu}, \mathrm{Fe}^{+2}$ and $\mathrm{Fe}^{+3}$, the most abundant heavy metals in the volcanic environment (Fig. 1d). Another important compound undergoing cross-feeding was the undecaprenyl-diphospho-N-acetylmuramoyl-(N-acetylglucosamine)-Lalanyl-D-glutamyl-meso-2,6-diaminopimeloyl-D-alanyl-D-alanine (uaagmda), which has an important role in peptidoglycan biosynthesis (KEGG ID C05898). The "uaagmda" showed a higher exchange rate in BR ( $0.79 \%$ vs $0.07 \%$ ), indicating greater cell wall biosynthesis in bacteria at low temperature. Studies of bacteria growing at low temperature revealed an increase amount of peptidoglycan (Mykytczuk et al., 2016; Tribelli and López, 2018), corroborating the results obtained herein based on flux balance analysis.

\section{Dynamicity and metabolic cooperation in the microbiome}

The correlation degree existing among abundance profiles of microbial species was calculated with methods suitable to analyze compositional data, followed by a network visualization approach (Fig. 3). Nodes in the network having a high correlation degree were investigated in more details since they can represent the most important interactions in complex systems (Pavlopoulos et al., 2011; Roume et al., 2015).

The reconstructed network includes two main clusters connected by a middle part (middle network section); a higher abundance of MAGs derived from BR in the left cluster, and of BG in the right one (Fig. 3a). Most MAGs (91\%) were positively correlated with at least another microbe. All Cyanobacteria were associated to the right cluster (3 MAGs), however only the two Pseudanabeana species showed a preferential association to BG (Fig. 3c). Previous studies performed with metagenomics or amplicon sequencing (16S rRNA) on biofilms reported low abundance of Cyanobacteria, despite the remarkable biological role this taxon has in the environment (Alvarenga et al., 2017; Anesio et al., 2017). It has to be considered that abundance is not always a good proxy for defining the importance of taxa in microbiomes, for this reason other methods, such as the identification of positive correlations or analysis of metabolic exchanges, are needed. For example, MAGs assigned to the Psychrobactergenus, one of the most abundant taxa found in previous metagenomic studies performed in Whalers Bay (Centurion et al., 2019; Centurion et al., 2021), did not show strong positive correlation with other MAGs and was excluded from this analysis.

Differently from Psychrobacter, the other two most abundant genera, Flavobacterium and Polaromonas $(\mathrm{n}=5$ each), showed a positive correlation with another MAG. Furthermore, 3 Flavobacterium spp. (WB_006, WB_121, WB_265) presented higher abundance in BR, while 2 of the Flavobacterium and Polaromonas genera were more abundant in BG (Fig. 3c), highlighting the high diverse taxonomic composition between the two clusters. Besides Flavobacterium, only the genera Lysobacter and 
Salinibacterium and the family Akkermansiaceae showed to have members in both clusters, all the others had preference for one of the two type of biofilm. This finding proves the marked effect of temperature and other physicochemical factors in shaping the microbiome composition on Whalers Bay sediments. For example, the mesophilic/thermophilic genus Porphyrobacter and the diazotrophic genus Hydrogenophaga were preferentially associated to the BG biofilm. This is corroborated by the BioEnv analysis (Best Subset of Environmental Variables with Maximum Rank), showing that the abiotic factors $\mathrm{Pb}$, temperature and nitrogen strongly influenced the microbiomes in WB biofilms (correlation of 0.31 ). However, only 45 out of 180 MAGs showed significant differences between the two types of biofilms, suggesting that $75 \%$ of MAGs display a different response to environmental stressors.

Because of the importance of nodes having multiple edges (defined as network "hubs") (Roume et al., 2015), MAGs have been selected according to their degree of interaction by using 20 edges as threshold. According to this, only seven MAGs for each cluster were selected for further consideration (Fig. 3b). All MAGs of the middle network section $(n=7)$ were also retained since they represent the connection between the two clusters. The MAGs identified as relevant were Alphaproteobacteria WB_595 (35 interactions) and Gemmatimonadaceae WB_43-12-0 (34 interactions) from the right cluster. These two hubs, together with other three MAGs having a high degree of correlations (Gemmatimonadetes_WB_603, Burkholderiales_WB_282 and Rhodobacterales_WB_585) formed a group of anoxygenic phototrophic bacteria (APB). The annotation of proteins encoded by APB revealed the presence of anoxygenic photosystem II genes (pufL and pufM) and the photosynthetic components of purple bacteria (puhA, pufC, pufA, pufB). Moreover, these APB MAGs have all the genes to produce photosynthetic pigments of phototrophic purple bacteria, the bacteriochlorophyll-A and B and chlorophyll-A from protoporphyrin IX (data not shown) (Scheer, 2004; Senge et al., 2004). The purple bacteria play an important role in the environment since they can degrade toxic compounds (mainly $\mathrm{H}_{2} \mathrm{~S}$ ), use non-fermentable organic compounds and fix nitrogen. According to these characteristics they are among the most metabolically versatile microorganisms (Madigan and Jung, 2009). Purple bacteria are able to grow in the absence of light, and this can represent an advantage in Antarctic environments where, during winter, long dark nights may last for weeks. Antarctic seasons can provide a key benefit for purple bacteria growth, which can support the survival of other microorganisms in the biofilm resulting in a high number of positive correlations among MAGs in the right cluster.

In the left cluster, the node correlation degree varies from 20 to 29 and the MAG having the highest degree is Pedobacter_WB_479 (29 interactions, ranking third among all MAGs). Differently from the marked prevalence of Proteobacteria and Gemmatimonadetes in the right cluster, the left one was characterized by a variety of different taxa cohexisting within the biofilm. Although Arthrobacter (Actinobacteria), Dokdonella (Proteobacteria), Pedobacter (Bacteroidetes) and Tissierellaceae (Firmicutes) have been previously reported as possibly involved in hydrocarbon degradation (Gutierrez et al., 2019; Prince et al., 2019), the annotation of MAGs related to these taxonomic groups did not show the presence of genes related to oil degradation process (e.g. alkB and nah) (Liu et al., 2015). The MAG WB_200 was assigned to the order Myxococcales_(Proteobacteria), that encompasses microorganisms capable of forming a 
fruiting body, a sporulation type with a relevant role during starvation and critical to face harsh environmental conditions (Huntley et al., 2010). The order Myxococcales comprises saprophytic microbes, typically found in surface environments and involved in decomposition of polymers in biofilms (Shimkets et al., 2006). Species belonging to Akkermansiaceae (Verrucomicrobia) were described in gut microbiome studies (Marques et al., 2016; Moraes et al., 2019). The family has only one genus (Akkermansia) and has not been previously reported in Antarctica or the Arctic. The MAGs assigned to Akkermansiaceae $(n=4)$ encode enzymes involved in degradation of carbohydrates, as arabinose, fucose, mixed-linkage glucans, rhamnose, starch, and xyloglucan (Supplementary Fig. 2).

All MAGs of the middle network section showed genes for obligatory or facultative anaerobic respiration. Anaerobic microorganisms are commonly reported in biofilms and usually grow in the internal part, where they are protected from the oxygen that is consumed by the aerobic microbes inhabiting the biofilm outer layers (Davey and O'Toole, 2000). Among the middle network MAGs, only the MAG

Bacteriovoracaceae_WB_212 showed potential for aerobic respiration. Members of this family have been previously reported as a predator of gram-negative bacteria (Davidov and Jurkevitch, 2004). Interestingly, Bacteriovoracaceae_WB_212 showed positive correlation with the gram-negative MAGs Paludibacter_WB_327 and Rhodoferax_WB_6.

In summary, the most abundant MAGs in the green biofilm (right cluster, Fig. 3b) included various autotrophic bacteria that need light to grow, but they also showed heterotrophic machinery to grow in the dark. On the other hand, the most abundant MAGs in the red biofilm (left cluster, Fig. 3a) included a group of bacteria, mostly aerobic gram-negative microbes, capable of using various organic compounds to grow at low temperatures. The middle network section, connecting the right and left clusters, included species which are probably growing in the internal part of both biofilms under the protection of the external aerobic bacteria.

\section{Active microbial species and expressed functions in the biofilms}

RNA-seq reads obtained for the sample collected in 2017 have been mapped to the MAGs in order to explore their functional activity. Genome-centric metatranscriptomics was performed only considering the relevant MAGs selected from the interaction network. TopGO analysis (Alexa and Rahnenfuhrer, 2020) queried all ORFs in the MAGs in order to visualize the Gene Ontology terms (GO; Ashburner et al., 2000; The Gene Ontology Consortium, 2018) associated to the genes with highest transcriptional activity. Only five MAGs of each cluster and the middle network section showed enrichment of GO terms with p-value lower than 0.05 (Fig. 4a-c). Nonetheless, the terms "growth", "translation" and other 28 terms associated to "metabolic process" and "division" ( 53\% of GO terms) were enriched in clusters BR, BG and in the middle cluster (Supplementary Table 4). These terms demonstrated that the selected MAGs are not dormant but they are actively growing in the biofilms. Despite the low temperature measured at the time of sampling, the activity is not surprising since the literature already reported that Antarctic bacteria can grow at temperatures as low as $-12^{\circ} \mathrm{C}$ (Breeze et al., 2004). 
In addition to the metabolic terms, 17 (32\%) were related to the response to environmental stressors (oxygen level, cold, and heat) and to toxic substances (chemical and reactive oxygen species - ROS). Interestingly, MAGs in the BR cluster and in the middle cluster seemed more actively involved in the response to "heat" and "hypoxia", while those in the BG seemed more responsive to "cold" and "heat". The responses to environmental stressors were related to the "growth and respiration" classification and suggested that BR cluster included more psychrophilic and obligate aerobic MAGs, the middle network section included more anaerobic and mesophilic/psychrophilic MAGs, while the BG cluster had more mesophilic MAGs with versatile respiration. The remaining $9 \%$ of the GO terms were related to the "transport of substances" (metal and proteins) and to the "locomotory" response to external stimuli; these terms were mainly represented in MAGs of the middle network section.

To better understand the relevance of different GO/KEGG terms in the MAGs associated to the three clusters, a differential binomial analysis with DESeq2 was made ( $p$-value $<0.05)$. MAGs of the middle network section did not show higher gene expression level in any of the biofilms, proving their versatility and ability to grow in both conditions. Regarding MAGs specifically associated to the biofilms (BR and BG clusters), those having the highest number of differentially expressed genes were:

Dokdonella_WB_456, representative of BR, and Alphaproteobacteria_WB_595, representative of BG. Apart from the genes encoding metabolic enzymes, as previously mentioned, stress-related genes were also found differentially expressed, and are reported in Fig. 4d. The only expressed genes not having a direct connection with stress response, and representing the more striking difference between BG and BR MAGs, were the ones related to anoxygenic photosystem of purple bacteria present in Alphaproteobacteria_WB_595 of BG. This multimeric structure is composed by the light-harvesting core antenna 1 (LH1; gene pufAB) and 2 (LH2; gene pucAB), and the reaction center (RC; gene pufCL). In the pigments (chlorophyll and bacteriochlorophyll), the proteins of the antennas form a complex structure which is able to harvest photons and perform the energy transfer to the RC complexes (Saga et al., 2019).

The main functional group of genes differentially expressed in the two MAGs was represented by chaperons (beige color in Fig. 4d), which can transfer denatured proteins (HSP20, gene ibpA) to a folding system (genes $c / p \mathrm{~B}, f k p \mathrm{~A} / f k / \mathrm{B}, p r s \mathrm{~A}$ ) or to a proteolysis system (genes $c / p \mathrm{~A}, c / p \mathrm{C}$, lon). This process avoids the irreversible folding and/or eliminates unfolded outer membrane proteins (degP/pepP). The heat shock proteins (HSP), named the small HSP (HSP20; gene ibpA) and the HSP100 (gene clpABC), were previously reported in the literature as a machinery of paramount importance during the stress response in Antarctic environments (Campanaro et al., 2010; Centurion et al., 2021). The HSP100 are essential proteases for the maintanance of the cell homeostasis (Noor, 2015). Nonetheless, the HSP are not only linked with the response to the heat shock, but instead are a general system to cope with stress response to various molecules (toxic substances, oxidative stress, heavy metals) (Maleky et al.,2016). In this work, the sHSP and HSP100 were shown to have a central role in supporting the growth of Antarctic bacteria, since they remain active also at low temperature $\left(0^{\circ} \mathrm{C}\right)$ due to the stimulus operated by unfolded proteins. The $C / p C$-mediated (HSP100) response to low temperature was already reported in the literature, demonstrating its crucial role in maintaining the growth and the photosynthetic activity in Synechococcus 
PCC 7942 (Porankiewicz and Clarke, 1997). Moreover, the sediments in WB have high concentrations of heavy metals (Fig. 1d), and these are amongst the molecules stimulating the HSP response. Following this additional selective pressure in WB, the resistance gene counteracting the negative effects of copper and silver ( $\cos \mathrm{B})$ are amongst the most expressed efflux pump systems in Alphaproteobacteria_WB_595. As an additional mechanism to block the entrance of toxic compounds (antibiotic and small chemicals), Dokdonella_WB_456 also expresses the ompAF-encoded porin channel (Chou and Lee, 2019). In Alphaproteobacteria_WB_595, the multidrug efflux pump acrA exports antibiotics and biocides from the cytoplasm to the external environment. Furthermore, the periplasmic defense enzyme cytochrome- $\mathrm{C}$ peroxidase $(c c p)$ can degrade toxic peroxides $\left(\mathrm{H}_{2} \mathrm{O}_{2}\right)$ in the absence of oxygen (Khademian and Imlay, 2017).

The membrane proteins encoded by genes cirA/fhuA, exbB/to/Q and exbD/to/R are part of the TonBdependent transporters (TBDTs family), which are involved in the uptake of iron and nickel complexes, vitamin B12, and carbohydrate to the cytoplasm (Noinaj et al., 2010). Nonetheless, the TBDTs are the gateway of bacteriocins, more specifically the colicins A and B, produced by bacteria exposed to stressful conditions (Buchanan et al., 2007). Thereby, results gathered in the present study demonstrated that Antarctic bacteria are constantly exposed to environmental stressors. In fact, the genes identified as differentially expressed are involved in resistance to ROS, heavy metals, and toxic compounds, and in the maintenance of cellular homeostasis through the degradation of unfolded proteins or supporting protein folding.

\section{Conclusions}

Both types of biofilms sampled from WB sediments, in Deception Island, are formed by a high complex microbiome, with composition strongly influenced by light exposure and temperature. Despite the recovered MAGs were related to taxa of marine origin, a small number was previously described revealing a high degree of novelty in the genomes. This finding suggests that an enormous number of species present in the oceanic environment remains still unknown at the genome level and they deserve a large effort in the next future to allow a comprehensive understanding of the marine microbiome. The two biofilms showed remarkable differences: because of the light exposure and the higher temperature, BG harbors autotrophic microorganisms and other species that are not exclusively psychrophilic and psychrotolerant, while microbes in BR are capable of growing at lower temperatures and are also involved in the degradation of complex substrates. The analysis of the correlation degree measured between MAGs provided a new perspective on complex microbiological systems and allowed the identification of crucial members of the biofilm independently on their abundance. MAGs associated with purple bacteria in $B G$ presented the highest numbers of interactions with other species, in particular the Alphaproteobacteria_WB_595, which seems to have a crucial metabolic role in the biofilm and deserves a deeper investigation in future studies. The metatranscriptomic analysis allowed to gain insights on MAGs activity at genome-centric level, revealing an active grow. Finally, the chaperon system and the resistance genes counteracting the negative effect of ROS and toxic compounds are essential for maintaining the 


\section{Declarations}

\section{Ethics approval and consent to participate}

Not applicable

\section{Consent for publication}

Not applicable.

\section{Availability of data and material}

Metagenomic sequences were submitted to the European Nucleotide Archive (http://www.ebi.ac.uk) under the project accession number PRJEB29861 for 2014 samples and PRJEB38669 for 2015 and 2017 samples. Metatranscriptomic sequences were submitted under the project accession number PRJEB42062.

\section{Competing interests}

The authors declare that they have no competing interests

\section{Funding}

This work was supported by the São Paulo Research Foundation - FAPESP (Processes no. 2016/05640-6, 2017/03172-8 and 2019/22891-0).

\section{Authors' contributions}

VBC conceptualization, designed the strategy for sampling, prepared DNA and RNA for sequencing, designed the strategy for metagenomic and metatranscriptomic data analysis, analyzed metagenomic and metatranscriptomic data, and drafted the manuscript; SC designed the strategy for metagenomic and metatranscriptomic data analysis, analyzed metagenomic and metatranscriptomic data and revised the manuscript; AB Flux balance analysis, and revised the manuscript; LT strategy for metagenomic and metatranscriptomic data analysis, and revised the manuscript VMO conceptualization, designed and supervised experiments, and revised the manuscript. 


\section{Acknowledgments}

The authors are grateful to the São Paulo Research Foundation - FAPESP (Processes no. 2016/05640-6, 2017/03172-8 and 2019/22891-0), the Proantar - Brazilian Antarctic Program and the Project MycoAntar diversity and bioprospecting of Antarctic fungi.

\section{References}

Alexa A, Rahnenfuhrer J (2020). topGO: Enrichment Analysis for Gene Ontology. R package version 2.40.0.

Alneberg, $\mathrm{J}$ et al. Binning metagenomic contigs by coverage and composition. Nature Methods. 11, 11441146 (2014). doi:10.1038/nmeth.3103

Alvarenga, DO; Fiore, MF; Varani, AM. A metagenomic approach to cyanobacterial genomics. Frontiers of Microbiology. 8, 809 (2017). doi:10.3389/fmicb.2017.00809

Anesio, AM et al. The Microbiome of glaciers and ice sheets. NPJ Biofilms and Microiomes. 3, 10 (2017). doi:10.1038/s41522-017-0019-0

Ashburner, M et al. Gene Ontology: tool for the unification of biology. Nat genet. 25, 25-29 (2000). doi:10.1038/75556

Asnicar, $\mathrm{F}$ et al. Precise phylogenetic analysis of microbial isolates and genomes from metagenomes using PhyloPhIAn 3.0. Nature Communications. 11, 2500 (2020). https://doi.org/10.1038/s41467-02016366-7

Bartolini, S; Geyer, A; Martí, J; Pedrazzi, D; Aguirre-Díaz, G. Volcanic hazard on Deception Island (South Shetland Islands, Antarctica). Journal of Volcanology and Geothermal Research. 285, 150-168 (2014) http://dx.doi.org/10.1016/j.jvolgeores.2014.08.009

Basile, A et al. Revealing metabolic mechanisms of interaction in the anaerobic digestion microbiome by flux balance analysis. Metabolic Engineering. 62, 138-149 (2020). https://doi.org/10.1016/j.ymben.2020.08.013

Bej, AK; Aislabie, J; Atlas, RM. Polar Microbiology: The Ecology, Biodiversity, and Bioremediation Potential of Microorganisms in Extremely Cold Environments. 424 (CRC Press, 2009). https://doi.org/10.1201/9781420083880

Bendia, AG et al. A Mosaic of Geothermal and Marine Features Shapes Microbial Community Structure on Deception Island Volcano, Antarctica. Frontiers in Microbiology, 9, 899 (2018a). doi:10.3389/fmicb.2018.00899 
Bendia, AG et al. Surviving in hot and cold: psychrophiles and thermophiles from Deception Island volcano, Antarctica. Extremophiles, 22, 917-929 (2018b). https://doi.org/10.1007/s00792-018-1048-1

Bendia, AG et al. Metabolic potential and surivival strategies of microbial communities across extreme temperature gradients on Deception Island volcano, Antarctica. BioRxiv preprint. doi:

https://doi.org/10.1101/2020.08.07.241539

Bolger, AM; Lohse, M; Usadel, B. Trimmomatic: A flexible trimmer for Illumina Sequence Data.

Bioinformatics. 30, 2114-2120 (2014). https://doi.org/10. 1093/bioinformatics/btu170

Breezee, J; Cady, N; Staley, JT. Subfreezing growth of the sea ice bacterium "Psychromonas ingrahamii". Micriobial Ecol. 47, 300-304 (2004). doi:10.1007/s00248-003-1040-9

Buchanan, SK. Structure of colicin I receptor bound to the R-domain of colicin la: implications for protein import. The EMBO Journal. 26, 2594-2604 (2007). doi:10.1038/sj.emboj.7601693

Buda, $\mathrm{J}$ et al. Biotope and biocenosis of cryoconite hole ecosystems on Ecology Glacier in the maritime Antarctic. Science of Total Environmental. 724 (2020). https://doi.org/10.1016/j.scitotenv.2020.138112

Cameron, KA; Hodson, AJ; Osborn, AM. Structure and diversity of bacterial, eukaryotic and archaeal communities in glacial cryoconite holes from the Arctic and the Antarctic. FEMS Microbiol. Ecol. 82, 254267 (2012).

Campanaro, $\mathrm{S}$ et al. Temperature-dependent global gene expression in the Antarctic archaeon Methanococcoides burtonii. Environmental Microbiology. 13, 2018-2038 (2010).

https://doi.org/10.1111/j.1462-2920.2010.02367.x

Centurion, VB et al. Unveiling resistome profiles in the sediments of an Antarctic volcnic island. Environmental Pollution. 255, 113240 (2019). doi:10.1016/j.envpol.2019.113240

Centurion, VB et al. Dynamics of microbial stress responses driven by abiotic changes along a temporal gradient in Deception Island, Maritime Antarctica. Science of Total Environmental. 758 (2021). https://doi.org/10.1016/j.scitotenv.2020.143671

Chaumeil, P; Mussig, AJ; Hugenholtz, P; Parks, DH. GTDB-TK: a toolkit to classify genomes with the genome taxonomy database. 36, 1925-1927 (2020). https://doi.org/10.1093/bioinformatics/btz848

Chou, U; Lee, C. Distinct roles of outer membrane porins in antibiotic resistance and membrane integrity in Escherichia coli. Frontiers in Microbiology. 10 (2019). doi:10.3389/fmicb.2019.00953

Clarke, KR; Ainsworth, M. A method of linking multivariate community structure to environmental variables. Marine Ecology Progress Series. 92, 205-219 (1993). doi:10.3354/meps092205 
Davey, ME; O’Toole, GA. Microbial Biofilms: from ecology to molecular genetics. Microbiology and Molecular Biology Reviews. 64, 847-867 (2000). doi:10.1128/MMBR.64.4.847-867.2000

Davidov, Y; Jurkevitch, E. Diversity and evolution of Bdellovibrio-and-like organisms (BALOs), reclassification of Bacteriovorax starrii as Peredibacter starrii gen. Nov., comb. Nov., and description of the Bacteriovorax-Peredibacter clade as Bacteriovoracaceae fam. Nov. International Journal of Systematic and Evolutionary Microbiology. 54, 1439-1452 (2004). doi:10.1099/ijs.0.02978-0

Delforno, TP et al. Comparative metatranscriptomics analysis of anaerobic digesters treating anionic surfactant contaminated wastewater. Science of Total Environmental. 649, 482-494 (2019). https://doi.org/10.1016/j.scitotenv.2018.08.328

Delmond, TO et al. Nitrogen-fixing populations of Plactomycetes and Proteobacteria are abundant in surface ocean metagenomes. Nature Microbiology. 3, 804-813 (2018). https://doi.org/10.1038/s41564018-0176-9

Downes, MT; Hrstich, L; Vincent, WF. Extraction of chlorophyll and carotenoid pigments from Antarctic benthic mats for analysis by HPLC. J Appl Phycol. 5, 623-628 (1993).

Duarte AWF, Centurion VB, De Oliveira, VM. Chapter 2: Uncultivated Fungi from Antarctica. In: ROSA LH, Fungi of Antarctica. Springer Nature Switzerland. (Springer, 2019). doi:10.1007/978-3-030-18367-7_2

El-Gebali, S et al. The Pfam protein families database in 2019. Nucleic Acids Research. 47, D427-D432 (2019). https://doi.org/10.1093/nar/gky995

Fontana, A et al. Microbial activity response to hydrogen injection in thermophilic anaerobic digesters revealed by genome-centric metatranscriptomics. Microbiome. 6:194 (2018).

https://doi.org/10.1186/s40168-018-0583-4

Friedman, J; Alm, EJ. Inferring correlation networks from genomics survey data.PloS Comput Biol. 8 (2012). doi:10.1371/journal.pcbi.1002687

Geyer, A et al. Deciphering the evolution of Deception Island's magmatic system. Scientific Reports. 9, 373 (2019). https://doi.org/10.1038/s41598-018-36188-4

Graham, ED; Heidelberg, JF; Tully, BJ. BinSanity: unsupervised clustering of environmental microbial assemblies using coverage and affinity propagation. PeerJ. 5 (2017). https://doi.org/10.7717/peerj.3035

Gutierrez, T. in: axonomy, genomics and ecophysiology of hydrocarbon-degrading microbes (eds Timmis, KN; Boll, M; Geiger, O; Goldfine, H; Krell, T; Lee, SY; McGenity, TJ; Rojo, F; Sousa, DZ; Stams, AJM; Steffan, RJ; Wikes, H.) 143-152 (Springer Nature, 2019). https://doi.org/10.1007/978-3-030-14796-9

Hoham, RW; Duval, B. in Snow Ecology: An Interdisciplinary Examination of Snow-Covered Ecosystems (eds Jones, HG; Pomeroy, J.W; Walker, D A; Hoham, RW) 168-228 (Cambridge University Press, 2001). 
Huerta-Cepas, J et al. EggNOG 5.0: a hierarchical, functionally and phylogenetically annotated orthology resource based on 5090 organisms and 2502 viruses. Nucleic Acids Res. 47, D309-D314 (2019). doi:10.1093/nar/gky1085

Huntley, S et al. Comparative genomic analysis of fruiting body formation in Myxococcales. Mol. Biol. Evol. 28, 1083-1097 (2011). doi:10.1093/molbev/msq292

Hyatt, D. et al. Prodigal: prokaryotic gene recognition and translation initiation site identification. BMC Bioinformatics, 11 (2010). doi:10.1186/1471-2105-11-119

Jeon, $\mathrm{CO}$ et al. Polaromonas naphthalenivorans sp. nov., a naphthalene-degrading bacterium from naphthalene-contaminated sediment. International Journal of Systematic and Evolutionary Microbiology. 54, 93-97 (2004). doi:10.1099/ijs.0.02636-0

Kanehisa, M; Goto, S. KEGG: Kyoto encyclopedia of genes and genomes. Nucleic Acids Research. 28, 2730 (2000). doi:10.1093/nar/28.1.27

Kang, DD et al. MetaBAT 2: an adaptive binning algorithm for robust and efficient genome reconstruction from metagenome assemblies. PeerJ. 7 (2019). doi:10.7717/peerj.7359

Kang, DD; Froula, J; Egan, R; Wang, Z. MetaBAT, an efficient tool for accurately reconstructing single genomes from complex microbial communities. PeerJ. 3 (2015). https://doi.org/10.7717/peerj.1165

Keely, BJ. In: Advances in Photosysnthesis and Respiration: Chlorophylls and Bacteriochlorophylls (eds Grimm, B; Porra, RJ; Rudiger, W; Scheer, H) 25, 536-557 (Springer, 2006)

Khademian, M; Imlay, JA. Escherichia coli cytochrome $c$ peroxidase is a respiratory oxidase that enables the use of hydrogen peroxide as a terminal electron acceptor. 114, E6922-E6931 (2017). https://doi.org/10.1073/pnas.1701587114

Lami, R; Cottrell, MT; Campbell, BJ; Kirchman, DL. Light-dependent growth and proteorhodopsin expression by Flavobacteria and SAR11 in experiments with Delaware coastal waters. Environmental Microbiology. 11, 3201-3209 (2009). doi:10.1111/j.1462-2920.2009.02028.x

Langmead, B; Salzberg, S. Fast gapped-read alignment with Bowtie 2. Nat. Methods. 9, 357-359 (2012). doi: 10.1038/nmeth.1923

Lee, GLY et al. Biodegradation of phenol by cold-adapted bacteria from Antarctic soils. Polar Biology. 41, 553-562 (2018). https://doi.org/10.1007/s00300-017-2216-y

Lee, JW. Protocol measuring horizontal gene transfer from algae to non-photosynthetic organisms. MethodsX. 6, 1564-1574 (2019). http://dx.doi.org/10.1016/j.gene.2019.03.014 
Letunic, l; Bork P. Interactive Tree Of Life (iTOL): an online tool for phylogenetic tree display and annotation. Bioinformatics 23(1), 127-8 (2006). doi:10.1093/bioinformatics/btl529

$\mathrm{Li}, \mathrm{H}$ et al. The sequence alignment/map format and SAMtools. Bioinformatics. 25, 2078-2079 (2009). https://doi.org/10.1093/bioinformatics/btp352

$\mathrm{Li}, \mathrm{H}$; Durbin, R. Fast and accurate short read alignment with Burrows-Wheeler transform. Bioinformatics. 25, 1754-1760 (2009). https://doi.org/10.1093/bioinformatics/btp324

Liu, Q et al. Distribution of petroleum degrading genes and factor analysis of petroleum contaminated soil from the Dagang Oilfield, China. Scientific Reports. 5 (2015). doi:10.1038/srep11068

Lombard, $V$ et al. The carbohydrate-active enzymes database (CAZy) in 2013. Nucleic Acids Research. 42, D490-D495 (2013). https://doi.org/10.1093/nar/gkt1178

Lonhienne, $T$ et al. Cloning Sequences, and characterization of two chitinase genes from the antarctic Arthrobacter sp. Strain TAD20: Isolation and partial characterization of the enzymes. Journal of Bacteriology. 183, 1773-1779 (2001). doi:10.1128/JB.183.5.1773-1779.2001

Love, Ml; Huber, W; Anders, S. Moderated estimation of fold change and dispersion for RNA-seq data with DESeq2. Genome Biology. 15, 550 (2014). doi:10.1186/s13059-014-0550-8.

Machado, D et al. Fast automated reconstruction of genome-scale metabolic models for microbial species and communities. Nucleic Acids Research. 46, 7542-4553 (2018).

https://doi.org/10.1093/nar/gky537

Madigan, MT; Jung, DO. In: The purple Phototrophic Bacter (eds Hunter, CN; Thurnauer, MC; Beatty, T) 115 (Springer Science 2009).

Maleki, F et al. Bacterial Heat Shock Protein activity. J Clin Diagn Res. 10, BE01-BE03 (2016). doi:10.7860/JCDR/2016/14568.7444

Margesin, R; Miteva, V. Diversity and ecology of psychrophilic microorganisms. Research in Microbiology. 162, 346-361 (2011). doi:10.1016/j.resmic.2010.12.004

Marques, TM et al. In: Gut microbiota and immune system-related diseases (eds Haller, D.) 261-291 (Springer Nature, 2018). doi:10.1007/978-3-319-90545-7

Mattes TE et al. The genome of Polaromonas sp. Strain JS666: Insights into the evolution of a Hydrocarbon and Xenobiotic-Degrading bacterium, and features of relevance to biotechnology. Applied and Environmental Microbiology. 74, 6405-6416 (2008). doi:10.1128/AEM.00197-08

Moraes, ACF; Almeida-Pittito, B; Ferreira, SRG. In: Microbiome and metabolome in diagnosis, therapy, and other strategic applications (eds Faintuch, J; Faintuch, S.) 393-400 (Elsevier, 2019). 
Mykytczuk, NCS et al. Microscopic characterizationof the bacterial cell envelope ofPlanococcus halocryophilus Or1 during subzero growth at $-15^{\circ} \mathrm{C}$. Polar Biol. 39, 701-712 (2016). https://doi.org/10.1007/s00300-015-1826-5

Noinaj, $\mathrm{N}$ et al. TonB-dependent transporters: regulation, structure, and function. Annu Rev Microbiol. 64, 43-60. doi:10.1146/annurev.micro.112408.134247

Noor, R.. Mechanism to control the cell lysis and the cell survival strategy in stationary phase under heat stress. SpringerPlus. 4 (2015). doi:10.1186/s40064-015-1415-7

Olm, MR; Brown, CT; Brooks, B; Banfield, JF. Drep: a tool for fast and accurate genomic comparisons that enables improved genome recovery from metagenomes through de-replication. The ISME Journal. 11, 2864-2868 (2017). https://doi.org/10.1038/ismej.2017.126

Pal, C; Bengtsson-Palme, J; Rensing, C; Kristiansson, E; Larsson, DGJ. BacMet: antibacterial biocide and metal resistance genes database, Nucleic Acids Res.42, D737-D743 (2014). doi: 10.1093/nar/gkt1252

Parks, $\mathrm{DH}$ et al. Assessing the quality of microbial genomes recovered from isolates, single cells, and metagenomes. Genome Research. 25, 1043-1055 (2014). doi:10.1101/gr.186072.114

Pavlopoulos, GA et al. Using graph theory to analyze biological networks. BioData Mining. 4 (2011). https://doi.org/10.1186/1756-0381-4-10

Pereira, S. et al. Complexity of cyanobacterial exopolysaccharides: composition, structures, inducing factors and putative genes involved in their biosynthesis and assembly. FEMS Microbiol. 33, 917-941 (2009). https://doi.org/10.1111/j.1574-6976.2009.00183.x

Porankiewicz, J; Clarke, AK. Induction of the heat shockprotein ClpB affects cold acclimation in the cyanobacterium Synechococcus sp. strain PCC 7942. J. Bacteriol. 179, 5111-5117 (1997). doi:10.1128/jb.179.16.5111-5117.1997

Prince, RC et al. In: Taxonomy, genomics and ecophysiology of hydrocarbon-degrading microbes (eds Timmis, KN; Boll, M; Geiger, O; Goldfine, H; Krell, T; Lee, SY; McGenity, TJ; Rojo, F; Sousa, DZ; Stams, AJM; Steffan, RJ; Wikes, H.) 1-39 (Springer Nature, 2019). https://doi.org/10.1007/978-3-030-14796-9

Quaggio, JA; Raij B. Comparação de métodos rápidos para a determinação da matéria orgânica em solos. Revistas Brasileira de Ciência do Solo, 3, 184-187 (1979).

Rajendhran, J; Gunasekaran, P. Strategies for accessing soil metagenome for desired applications. Biotechnology Advances. 26, 576-590 (2008). doi:10.1016/j.biotechadv.2008.08.002

Rawlings, ND et al. The MEROPS database of proteolytic enzymes, their substrates and inhibitors in 2017 and a comparison with peptidases in the PANTHER database. Nucleic Acids Research. 46, D624-D632 (2018). https://doi.org/10.1093/nar/gkx1134 
Remias, D. Cell Structure and Physiology of Alpine Snow and Ice Algae. (Springer, 2012).

Richter, I et al. Influence of soil properties on archaeal diversity and distribution in the McMurdo Dry Valleys, Antarctica. FEMS Microbiology Ecology. 89, 347-359 (2014). doi:10.1111/1574-6941.12322

Rogers, SO et al. Ecology of Subglacial Lake Vostok (Antarctica), Based on Metagenomic/Metatranscriptomic Analyses of Accretion Ice. Biology (Basel). 2, 629-650 (2013). doi:10.3390/biology2020629

Rossi, F; De Philippis, R. Role of Cyanobacterial exopolysaccharides in phototrophic biofilm and in complex microbial mats. Life. 5, 1218-1238 (2015). doi:10.3390/life5021218

Saga, $\mathrm{Y}$ et al. Selective oxidation of B800 bacteriochlorophyll a in photosynthetic light-harvesting protein LH2. Scientific Reports. 9 (2019). https://doi.org/10.1038/s41598-019-40082-y

Saul, DJ et al. Hydrocarbon contamination changes the bacterial diversity of soil from around Scott Base, Antarctica. FEMS Microbiology Ecology. 53, 141-155 (2005). doi:10.1016/j.femsec.2004.11.007

Scheer, H. In: Advances in Photosysnthesis and Respiration: Chlorophylls and Bacteriochlorophylls (eds Grimm, B; Porra, RJ; Rudiger, W; Scheer, H) 25, 1-26 (Springer, 2006)

Senge, MO; Wiehe, A; Ryppa, C. In: Advances in Photosysnthesis and Respiration: Chlorophylls and Bacteriochlorophylls (eds Grimm, B; Porra, RJ; Rudiger, W; Scheer, H) 25, 27-37 (Springer, 2006)

Shaffer, $M$ et al. DRAM for distilling microbial metabolism to automate the curation of microbiome function. BioRxiv preprint. https://doi.org/10.1101/2020.06.29.177501

Shannon, $\mathrm{P}$ et al. Cytoscape: a software environment for integrated models of biomolecular interaction networks. Genome Research. 13, 2498-504 (2003). doi: 10.1101/gr.1239303

Shimkets, L; Dworkin, M; Reichenbach, H. in: The prokaryotes (eds Dworkin, M; Falkow, S; Rosenberg, E; Schleifer, KH; Stackenbrandt, E.) 31-115 (Springer, 2006).

Sieber, CMK et al. Recovery of genomes from metagenomes via a dereplication, aggregation and scoring strategy. Nature Microbiology. 3, 836-843 (2018). https://doi.org/10.1038/s41564-018-0171-1

Subramaniam, K; Ahmad, SA; Shaharuddin, NA. Mini review on phenol biodegradation in Antarctica using native microorganisms. Aspac J. Mol. Biol. Biotechnol. 28, 77-89 (2020).

doi:10.35118/apjmbb.2020.028.1.08

The Gene Ontology Consortium. The Gene Ontology Resource: 20 years and still GOing strong. Nucleic Acids Res. 47, D330-D338 (2019). https://doi.org/10.1093/nar/gky1055

The UniProt Consortium. UniProt: a worldwide hub of protein knowledge. Nucleic Acids Research. 47, D506-D515 (2019). https://doi.org/10.1093/nar/gky1049 
Toseland, A; Moxon, S; Mock, T.; Moulton, V. Metatranscriptomes from diverse microbial communities: assessment of data reduction techniques for rigorous annotation. BMC Genomics. 15, 1-7 (2014). https://doi.org/10.1186/1471-2164-15-901

Tribelli, PM; López NI. Reporting key features cold-adapted bacteria. Life. 8 (2018). doi:10.3390/life8010008

Uetake, $\mathrm{J}$ et al. Microbial community variation in cryoconite granules on Qaanaaq Glacier, NW Greenland. FEMS Microbiology Escology. 92 (2016). doi:10.1093/femsec/fiw127

Van Goethem, M; Cowan, D. In: The ecological role of micro-organisms in the Antarctic environmental (ed Castro-Sowinski, S) 3-23 (Springer Polar Sciences, 2019). https://doi.org/10.1007/978-3-030-02786-5_1

Vázquez, S et al. Bacterial communities and chemical parameters in soils and coastal sediments in response to diesel spills at Carlini Station, Antarctica. Science of the Total Environment. 605-606, 26-37 (2017). https://doi.org/10.1016/j.scitotenv.2017.06.129

Vincent, WF. Evolutionary origins of Antarctic microbiota: invasion, selection and endemism. Antarctica Science. 12, 374-385 (2000). doi:10.1017/S0954102000000420

Watts, SC et al. FastSpar: rapid and scalable correlation estimation for compositional data. Bioinformatics. 35, 1064-1066. doi: 10.1093/bioinformatics/bty734

Wu, Y; Simonns, BA; Singer, SW. MaxBin 2.0: an automated binning algorithm to recover genomes from multiple metagenomic datasets. Bioinformatics. 32, 605-607 (2016).

https://doi.org/10.1093/bioinformatics/btv638

Yergeau, E; Kowalchuk, GA. Responses of Antarctic soil microbial communities and associated functions to temperature and freeze-thaw cycle frequency. Environmental Microbiology. 10, 2223-2235 (2008). doi: 10.1111/j.1462-2920.2008.01644.x

Zelezniak, A et al. Metabolic dependencies drive species co-occurrence in diverse microbial communities. PNAS. 112, 6449-6454 (2015). https://doi.org/10.1073/pnas.1421834112

Zhang, $\mathrm{H}$ et al. dbCAN2: a meta server for automated carbohydrate-active enzyme annotation. Nucleic Acids Research. 46, W95-W101 (2018). https://doi.org/10.1093/nar/gky418

\section{Figures}




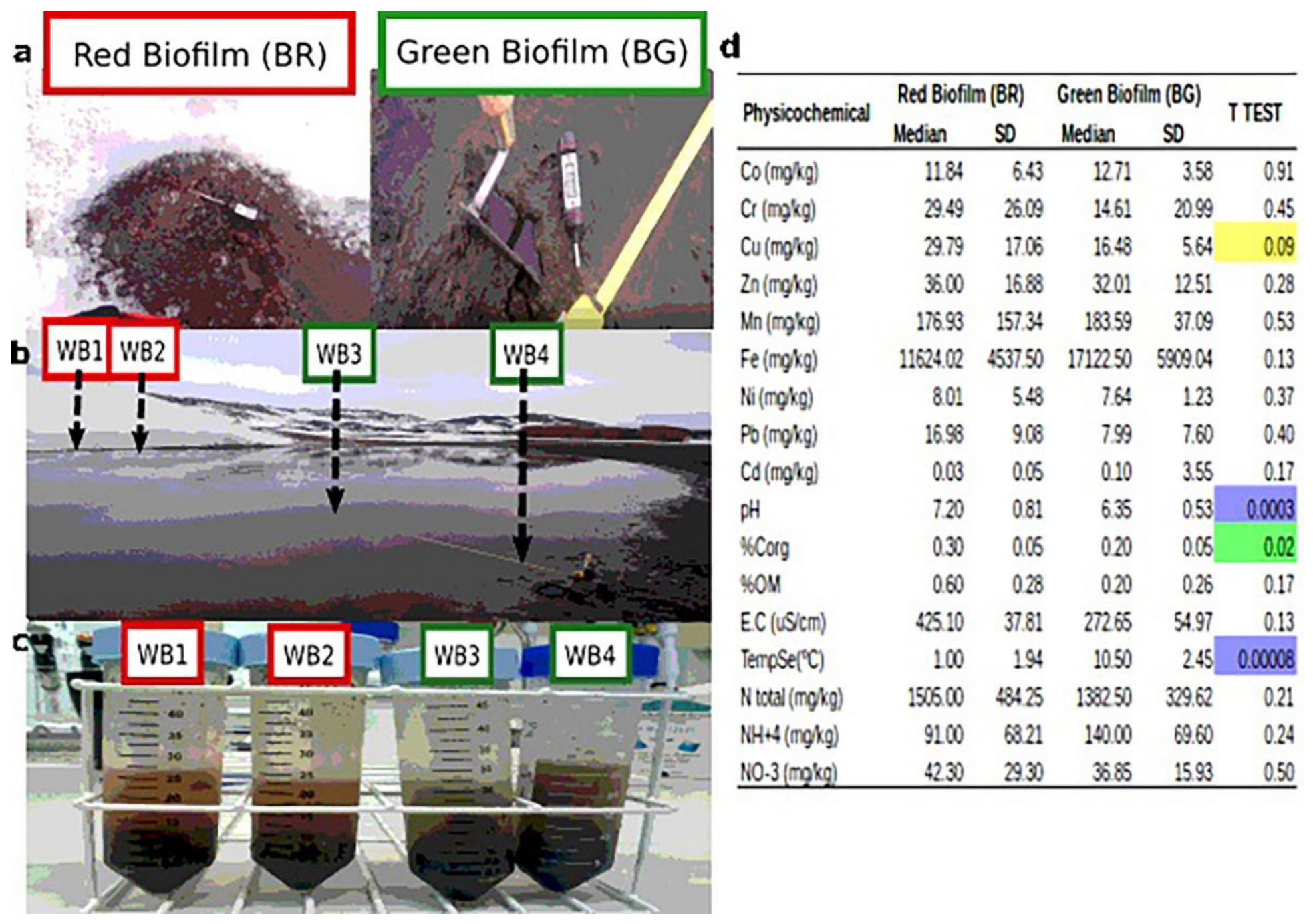

Figure 1

Biofilm characteristics. (a) Visual aspect of biofilms; (b) Sampling sites; (c) Falcon tubes containing samples for DNA extraction; (d) Physicochemical information of the biofilms with the median and standard deviation. The colors of $t$ test represents the $p$ values: yellow $p<0.01$; green $p<0.05$; blue $p$ $<0.01$. Corg: Organic Carbon; OM: Organic Matter; TempSe: Sediment Temperature $\left({ }^{\circ} \mathrm{C}\right) ; \mathrm{N}$ total: Total Nitrogen (mg/kg); NH+4: Ammonia (mg/kg); NO-3: Nitrate (mg/kg). 


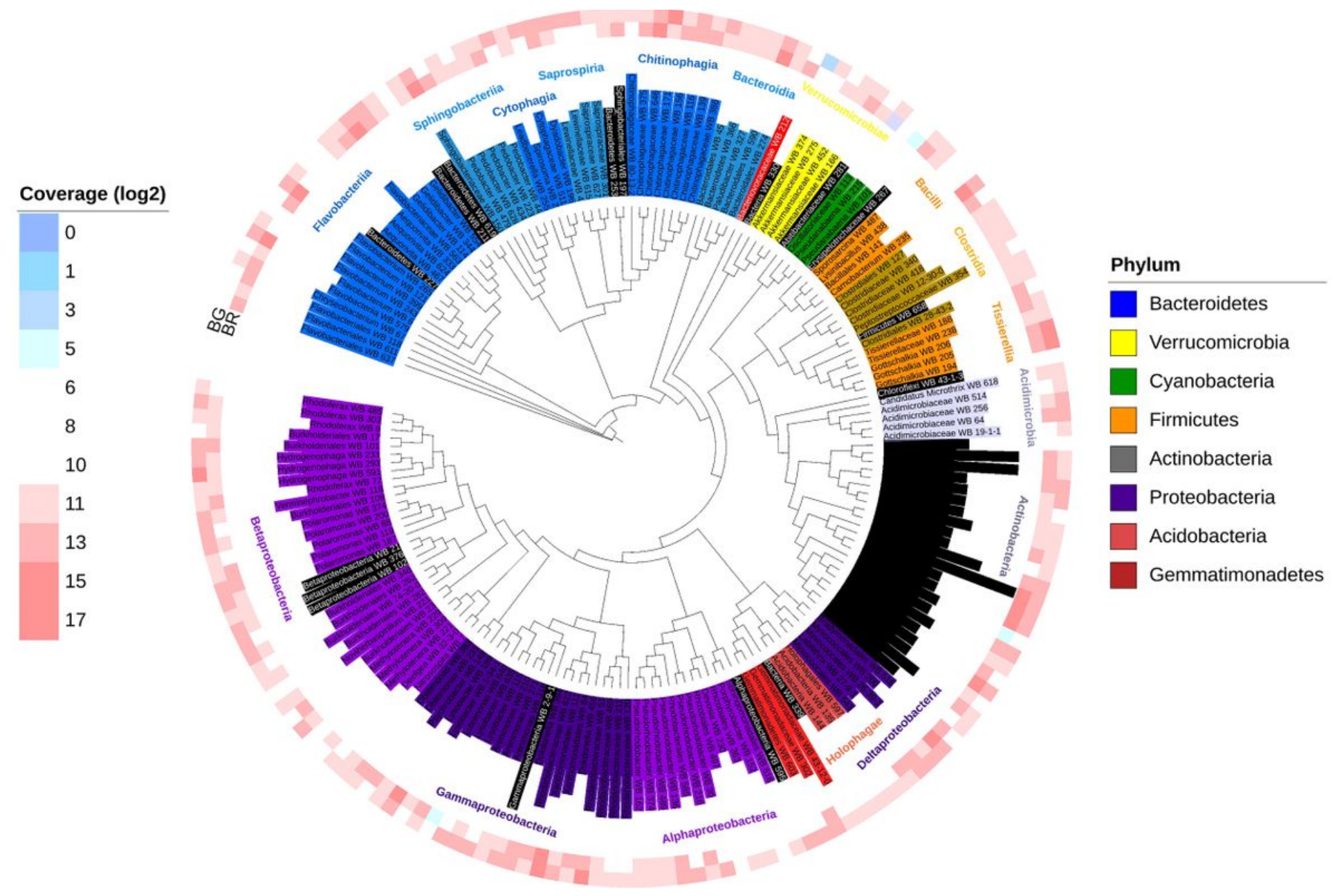

Figure 2

MAGs taxonomy. Tree of Phylophlan results where different colors represent the distinct phyla, and different shades of the same color represent the distinct classes. Black labeled-MAGs were classified only at phyla or kingdom levels. The red labeled-MAG is in the wrong place in the tree according to the taxonomic classification. The outer rings correspond to the abundance of BR and BG MAGs normalized by DESeq2. 


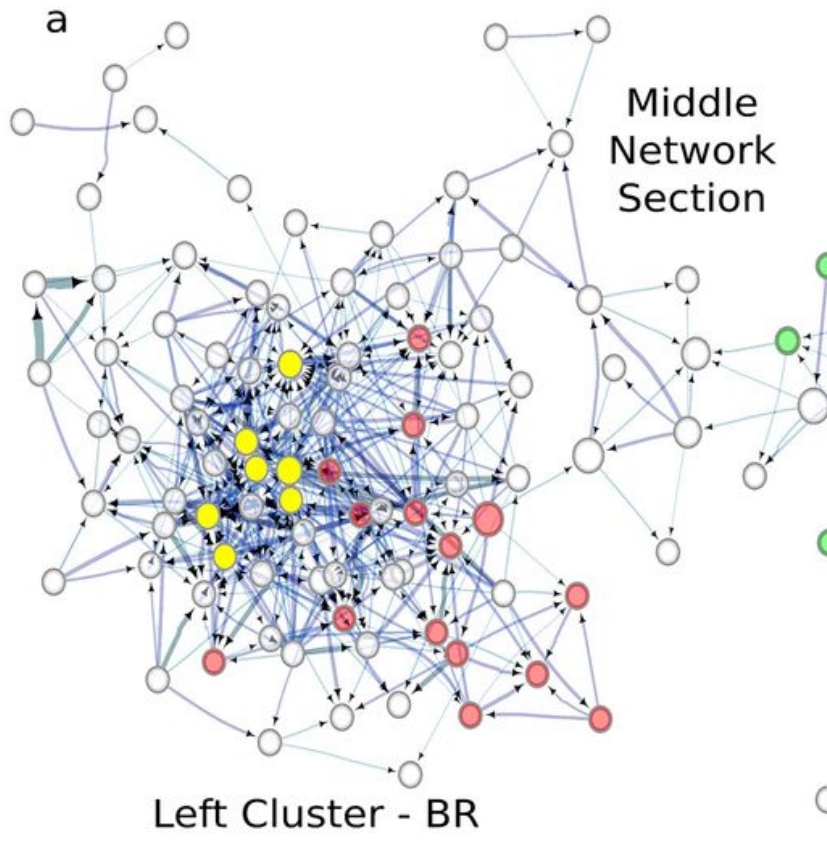

b

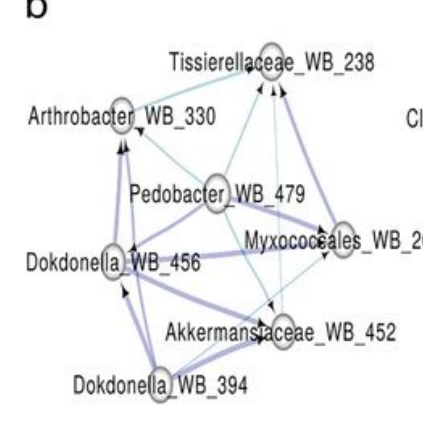

Selected Nodes
Burkholderiales_WB_17
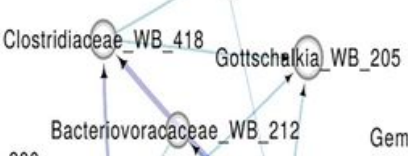

.

Rhodoferax =WB_6paludibacter WB 327

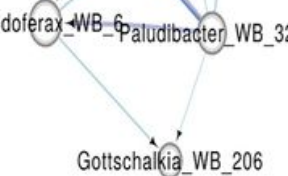

Gottschalikia__WB_206

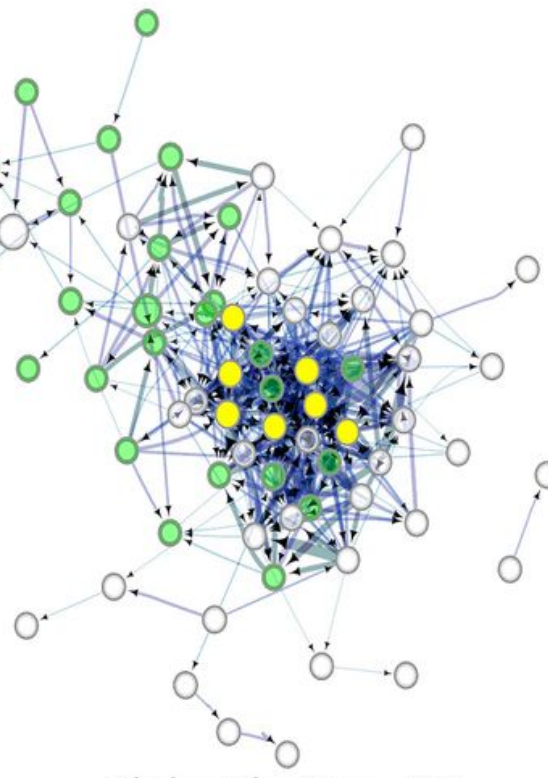

Right Cluster - BG

Burkholderales_WB_282

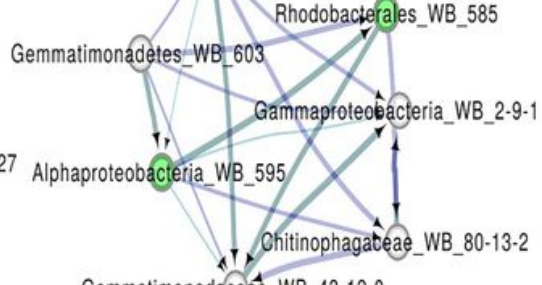

Gemmatimonadaceae_WB_43-12-0
C

\begin{tabular}{|c|c|c|}
\hline & & \\
\hline Acidimicrobiaceae_WB_514- & -3.49 & \\
\hline Akkermansiaceae_WB_166- & 2.39 & \\
\hline Akkermansiaceae_WB_374- & -5.1 & \\
\hline Alphaproteobacteria_WB_595- & -4.61 & \\
\hline Arthrobacter_WB_240- & 3.21 & \\
\hline Bacillales_WB_141- & 4.17 & \\
\hline Bacteria_WB_330- & 4.64 & \\
\hline Bacteroidetes_WB_227- & -3.15 & \\
\hline Betaproteobacteria_WB_376- & -3.18 & \\
\hline Burkholderiales_WB_282- & -3.52 & \\
\hline Chloroflexi_WB__43-1-3- & -4.35 & \\
\hline Clostridiāes_WB_127- & 2.92 & \\
\hline Cryobacterium_WB_201- & 2.17 & \\
\hline Devosia_WB_230- & -3.51 & \\
\hline Flavobacterium_WB_006- & 3.16 & \\
\hline Flavobacterium_WB_121- & 3.05 & \\
\hline Flavobacterium_WB_243- & -4.45 & \\
\hline Flavobacterium_WB_265- & 2.9 & \\
\hline Flavobacterium_WB_399- & -4.25 & log2FoldChange \\
\hline Hydrogenophaga_WB_233- & -3.08 & genter \\
\hline Hydrogenophaga_WB_293- & -3.06 & 2.5 \\
\hline Hydrogenophaga_WB_591- & -3.13 & \\
\hline Leadbetterella_WB_255- & -2.88 & 0.0 \\
\hline Lysobacter_WB_115- & -5.78 & -2.5 \\
\hline Lysobacter_WB_1-34-1- & 3.96 & \\
\hline Lysobacter_WB_635- & -7.55 & -5.0 \\
\hline Methylotenera_WB_221- & 3.8 & -7.5 \\
\hline Micrococcales_WB_355- & -7.61 & \\
\hline Microterricola_WB_371- & 3.28 & \\
\hline Nigerium_WB_43-10-2- & -3.47 & \\
\hline Oblitimonas_WB_231- & 3.25 & \\
\hline Pedobacter_WB_434- & -3.13 & \\
\hline Polaromonas_WB_200- & -5.7 & \\
\hline Polaromonas_WB_374- & -2.6 & \\
\hline Porphyrobacter_WB_152- & -5.84 & \\
\hline Pseudanabaena_WB_101 - & -5.44 & \\
\hline Pseudanabaena_WB_215- & -5.68 & \\
\hline Pseudorhodobacter_WB_30- & -3.62 & \\
\hline hodanobacteraceae_WB_120- & -3.95 & \\
\hline Rhodanobacteraceae_WB_51- & -3.02 & \\
\hline Rhodobacterales_WB_585- & -4.1 & \\
\hline Salinibacterium_WB_16-15-1. & 2.92 & \\
\hline Salinibacterium_WB_232- & -3.51 & \\
\hline Sphingomonadales_WB_616- & -4.74 & \\
\hline Tissierellaceae_WB_188- & 2.9 & \\
\hline & 2vs BG & \\
\hline
\end{tabular}

\section{Figure 3}

Cytoscape model and differential abundance of MAGs. (a) Interaction network with 163 MAGs positive correlated $(p<0.01)$. Yellow nodes correspond to the MAGs with more edge correlations. Red and Green nodes are the MAGs differentiated abundant of DESEq2 analyses and represent the biofilms BR and BG, respectively. (b) Interaction network of selected nodes (Yellow nodes in "a"). Green nodes are the MAGs differentiated abundant of DESEq2 analyses. (c) Heatmap based on the binomial differential analysis of DESeq2 $(p<0.05)$. 

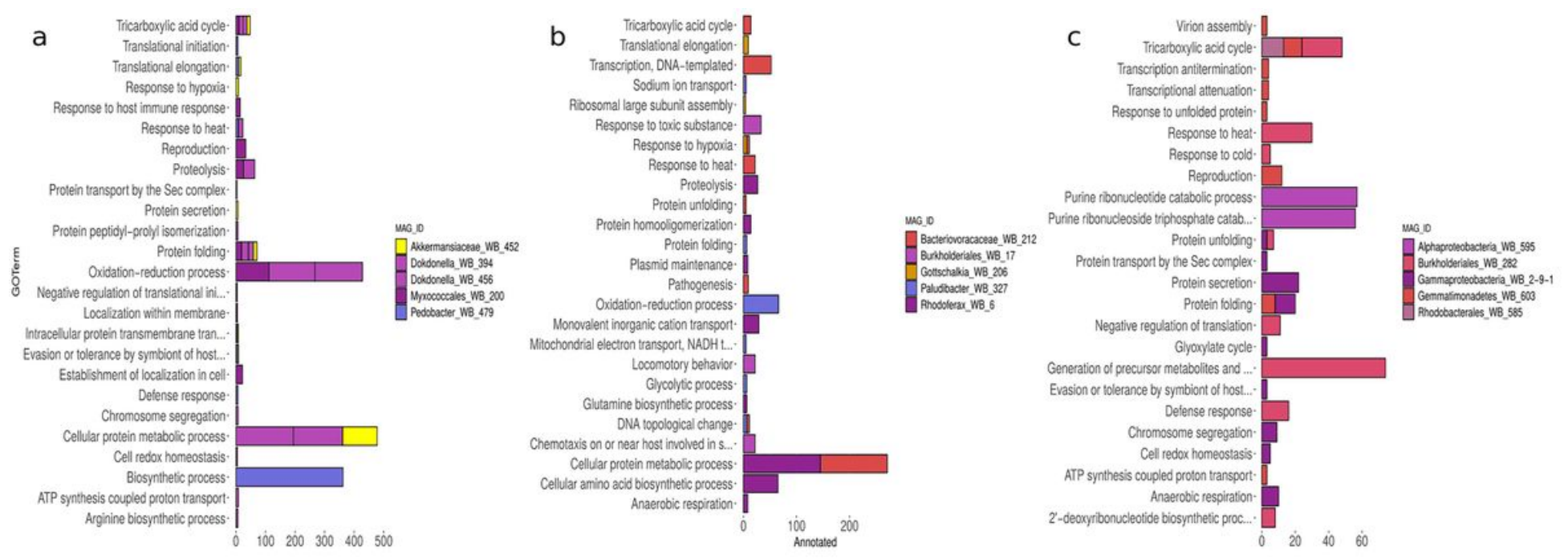

d

Dokdonella_WB_456

Alphaproteobacteria_WB_595
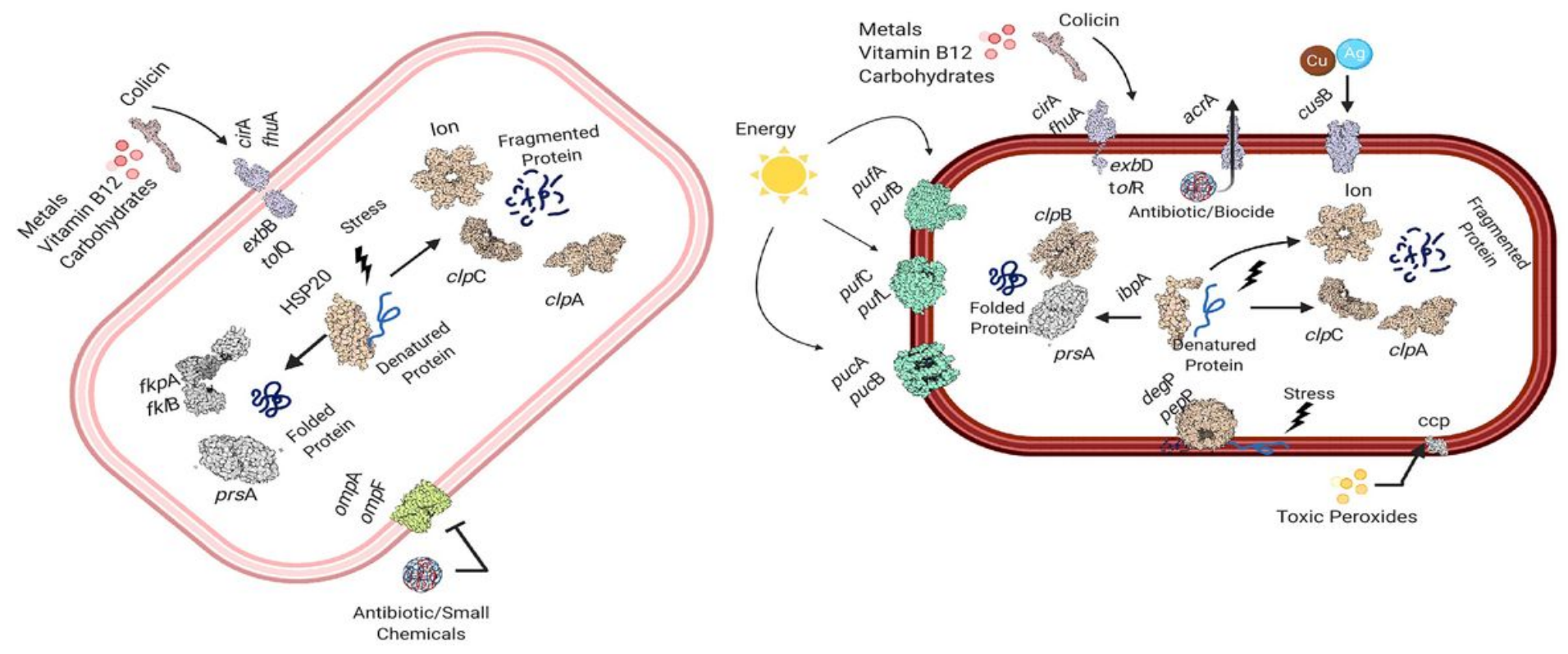

Figure 4

Metatranscriptomic Analysis. (a - c) Barplots of the enrichment analysis of topGO; (a) MAGs of the left cluster (BR), (b) the middle network section and (c) MAGs of the right cluster (BG). The barplots have only the terms of the "Biological Process" (BP) category, with p-value < 0.05. The Biological Process (BP) terms "growth" (G0:0040007) and "translation" (G0:0006412) were excluded from the analysis to facilitate the visualization of the remaining $53 \mathrm{GO}$ categories. (d) Genes of binomial differential analysis of DESeq2 ( $p$ < 0.05) of Dokdonella_WB_456 representing BR and Alphaproteobacteria_WB_595 representing BG. The protein colors represent the components of the cell, being: Beige, chaperons; purple, membrane transporters; gray, enzymes; lime green, porins; green: photosynthetic system.

\section{Supplementary Files}


This is a list of supplementary files associated with this preprint. Click to download.

- SupplementaryFigure1ClusteringDendrogramDREP.pdf

- SupplementaryFigure2heatmapDRAM.pdf

- SupplementaryTable1BinningStatistic.xlsx

- SupplementaryTable2MAGsCoverage.xlsx

- SupplementaryTable3SmetanaResults.xlsx

- SupplementaryTable4GOtermsinformation.xlsx 